\title{
The role of neutrophil to lymphocyte ratio in patients with pTa non-muscle invasive bladder cancer
}

\author{
Orsolya Mártha ${ }^{1}$, Daniel Balan ${ }^{1 *}$, Daniel Porav-Hodade ${ }^{1}$, Emőke Drágus ${ }^{1}$, Mihai \\ Dorin Vartolomei ${ }^{2}$, Călin Bogdan Chibelean ${ }^{1}$, Angela Borda ${ }^{3}$, Ákos Pytel ${ }^{4}$, Olivér \\ Árpád Vida ${ }^{1}$
}

1. Department of Urology, George Emil Palade University of Medicine, Pharmacy, Science and Technology of Targu Mures, Romania

2. Department of Cell and Molecular Biology, George Emil Palade University of Medicine, Pharmacy, Science and Technology of Targu Mures, Romania

3. Department of Histology, George Emil Palade University of Medicine, Pharmacy, Science and

Technology of Targu Mures, Romania

4. Department of Urology, University of Pécs, Hungary

\begin{abstract}
Introduction: The peritumoral inflammatory reaction has a substantial importance in the oncologic outcome of bladder cancer $(B C)$. One biomarker proven to be practical and accessible is the NLR (neutrophil-to-lymphocyte ratio) for high risk non-muscle invasive bladder cancer (NMIBC). The aim of the study was to investigate the role of NLR as a prognostic biomarker for disease recurrence, progression and survival of p Ta (pathological assesment of the primary tumor) NMIBC. Material and Methods: In our retrospective study we included 54 patients with pTa NMIBC from a total of 235 patients who underwent transurethral resection of bladder tumor (TURBT) during two consecutive years: January 2007 - December 2008 [median follow-up 106 months (interquartile range-IQR 68-116)]. Criteria for inclusion were: primary tumor, low-grade, with NLR available at 2 weeks prior to TURBT. $N L R$ was considered altered if higher than 3. Results: The median age of the patients included was 63 years (IQR 55 - 72). Most of the patients had NLR<3 (37 patients). Median EORTC (European Organization of Research and Treatment of Cancer) Recurrence Score was 4 (IQR 1-6), while EORTC Progression Score was 3 (IQR 0-6), respectively. Recurrence occurred in 8 out of 54 (14.81\%) patients and progression was identified in 2 out of 54 (3.70 $\%$ patients with muscle-invasive BC during follow-up. NLR $>3$ was not associated with clinical and pathological factors. In multivariable Cox regression analyses NLR as a continuous variable was an independent predictive factor for recurrence. Recurrence-free survival (RFS) Kaplan-Meier analysis did not show a statistical significance between NLR groups: $82.67 \%$ vs. 64.12\%, $p=0.26$. Kaplan-Meier analysis showed a lower Progression-free survival (PFS) in the NLR>3 group: $94.12 \%$ vs. 100\%, $p=0.04$. During follow-up (106 months) 18 patients deceased with
\end{abstract}

*Corresponding author: Department of Urology, University of Medicine, Pharmacy, Science and Technology of Targu Mures, Romania. E-mail: balan_dani@yahoo.com 
no impact of NLR as a prognostic factor in multivariable analyses. Kaplan-Meier overall survival (OS) analysis showed a 10-year OS of $70.27 \%$ in the low NLR group compared with $58.82 \%$ in the high NLR group, $p=0.45$. Conclusion: In this cohort, high NLR was associated with high recurrence rate in patients with Ta NMIBC. In low-risk NMIBC NLR could represent a valid biomarker for clinical usage regarding the intensity of follow-up schedule.

Keywords: neutrophil-to-lymphocyte ratio, non-muscle invasive bladder cancer, transurethral resection of bladder tumor, recurrence-free survival, progression-free survival

Received: 12 ${ }^{\text {th }}$ August 2019; Accepted: 30 ${ }^{\text {th }}$ October 2019; Published: $21^{\text {st }}$ November 2019

\section{Introduction}

Globally, bladder cancer (BC) is ranked the 9th most common of all diagnosed cancers. In 2018 alone, there were 550,000 new cases diagnosed and almost 200,000 deaths (1). In more developed countries, BC has a higher incidence (2) and this increases with age (50-70 years of age being the most affected group) and is three times more frequent in males than females (1).

At the time of detection, $75 \%$ of bladder tumors resulted in non-muscle-invasive bladder cancer (NMIBC), with a staged percentage distribution of $70 \%$ Ta stage, $20 \% \mathrm{~T} 1$, and $10 \%$ CIS (in situ carcinoma) $(3,4)$. The prognosis of NMIBC is favorable, however, $50-70 \%$ of the cases recur, with $10-20 \%$ developing into muscle-invasive bladder cancer (MIBC) over time. In terms of survival, the five-year overall survival (OS) rate reaches up to $88 \%(5,6)$. In high-risk NMIBC, the rate of five-year recurrence reaches $80 \%$, and progression can occur in up to $50 \%$ of all patients (7-9). These statistics make it one of the most costly cancers due to the lengthy follow-up procedures (5).

Tumor cells are surrounded by an inflammatory environment which is induced by the tumor or tumor-associated stroma. These are produced by chemokines, inflammatory mediators and significant quantities of immune cells in the tumor microenvironment. Inflammatory factors might promote the development and progression of the tumor (10). Increased laboratory values (neutrophils, $\mathrm{C}$ reactive protein) as a result of inflammatory processes may be predictive factors for tumor invasiveness or aggressiveness (11). The most studied biomarker of inflammation is the neutrophil-lymphocyte ratio (NLR), which was shown to be a prognostic factor in pancreas, breast, colon neoplasm (12) and tumors of urological origin, such as renal cell carcinoma (13) and urothelial carcinomas (14-17). Thus, there has been no focus on low-risk NMIBC. However, NLR is accepted as preoperative predictive factor for upper tract urothelial carcinoma according to the latest EAU guideline (18).

Thus, the aim of this study is to investigate NLR as a prognostic factor for recurrence, progression and survival of $\mathrm{p} \mathrm{Ta}$ (pathological assesment of the primary tumor) NMIBC.

\section{Material and Methods}

\section{Study Design}

We retrospectively reviewed 54 histologically confirmed pTa NMIBC patients who underwent transurethral resection of the bladder tumor (TURBT) between January 2007 and December 2008, with a median follow-up of 106 months (IQR 68-116). Multiple data were collected from the patients: age, gender, time of resection, recurrence, progression, tumor size, location, number of tumors, grade, stage. The study took place at the Urology Clinic of Târgu-Mureş and was approved by Mures County Hospital Ethical Committee. The last follow-up was completed in December 2018. Inclusion criteria were: primary tumor; low grade (G1/G2); NLR determination two weeks prior resection, and no adjuvant intravesical therapy following TURBT. NLR was 
considered altered if NLR was higher than three, as validated for HG (high-grade) T1 NMIBC (19).

\section{Histological Classification}

Histological classification was performed using both the 1973 World Health Organization classification and 2004 WHO classification of bladder tumors (20).

\section{Follow-up}

The clinical follow-up was based upon cystoscopy examination at three months in the initial two years, followed by cystoscopy at six months over the next three years and annually thereafter. Follow-up CT imaging was performed when clinically indicated, following EAU (European Association of Urology) guidelines at that time (21). The European Organization for Research and Treatment of Cancer (EORTC) scoring system and risk tables were used allowing estimation of the probability of progression and recurrence of the tumors. These calculations were based on: number of tumors, tumor size, prior recurrence rate, $\mathrm{T}$ category, concomitant CIS (in situ carcinoma) and grade (3).

Recurrence was defined as disease recurrence at more than 3 months after the initial intervention and progression was defined as the evolution of tumors to MIBC (Muscle Invasive Bladder Cancer). RFS and PFS were defined as the time from surgery to the first of either recurrence or relapse/progression or death $(22,23)$.

\section{Statistical Analysis}

Data were labeled as either nominal or quantitative variables. Nominal variables were characterized using frequencies. Quantitative variables were tested for normality of distribution by applying the Kolmogorov-Smirnov test and were described by mean \pm standard deviation or median and quartiles. The frequencies of nominal variables were compared with the chi-square test. Survival analysis was performed using the Kaplan-Meyer method, and the log-rank test was used for univariate comparisons.

We performed multivariate Cox proportional hazards regression analyses and used backward stepwise elimination to distinguish insignificant co-factors from significant predictors, we included in the multivariate analysis standard prognostic factors age, gender (male vs female), diameter ( $<3 \mathrm{~cm}$ vs $\geq 3 \mathrm{~cm})$, grade (G1 vs G2) and multifocality (Single vs Multiple) and NLR (continuous and NLR $<3$ vs. NLR $\geq 3$ ).

Harrell's concordance index (c-index) with its $95 \%$ confidence intervals $(\mathrm{CI})$ was used to measure the ordinal predictive power of the model (age, gender, diameter, grade, multifocality and NLR) for RFS and OS (24). All P values were two-sided and statistical significance was defined as a $\mathrm{P}<.05$. Statistical analyses were performed using Stata 11.0 statistical software (Stata Corp., College Station, TX).

\section{Results}

The median age of the patients was 63 years (IQR 55-72). In a significant proportion of the individuals, we identified NLR $<3$ (37 patients/68.51\%). The median recurrence EORTC (European Organization of Research and Treatment of Cancer) score was 4 (IQR 1-6) and the progression EORTC score was 3 (IQR $0-6)$. In total, $8(14.81 \%)$ patients experienced recurrence and $2(3.70 \%)$ patients revealed tumor progression during the follow-up period (at least pT2 stage) (29, IQR 25-36 months). NLR> 3 was not associated with other clinical and pathological factors. All patients with progression, however, were in the NLR $>3$ group (Table I). Utilization of the Kaplan-Meier analysis showed lower Progression-free survival (PFS) in the NLR> 3 group: $94.12 \%$ vs. $100 \%, p=0.04$ (Figure 1). In the low NLR group, 4 (10.8\%) patients had recurrence; likewise, $4(23.5 \%)$ pa- 
Table I. Characteristics of the 54 patients with pTa bladder cancer at diagnosis according to NLR value

\begin{tabular}{|c|c|c|c|c|}
\hline Patients characteristics & All patients & NLR $<3$ & NLR $>3$ & p value \\
\hline Total, n (\%) & 54 & 37 & 17 & \\
\hline Gender & $10(18.5 \%)$ & $6(162 \%)$ & $4(235 \%)$ & \multirow[b]{2}{*}{0.52} \\
\hline female & $44(81.5 \%)$ & $31(83.8 \%)$ & $13(76.5 \%)$ & \\
\hline \multirow{3}{*}{$\begin{array}{l}\text { Tu. Diameter }<3 \mathrm{~cm} \\
>3 \mathrm{~cm} \\
\text { Tu. multifocality } \text { single }\end{array}$} & $21(38.9 \%)$ & $12(32.4 \%)$ & $9(52.9 \%)$ & \multirow{2}{*}{0.15} \\
\hline & $33(61.1 \%)$ & $25(67.5 \%)$ & $8(47.1 \%)$ & \\
\hline & $24(44.4 \%)$ & $17(45.9 \%)$ & $7(41.2 \%)$ & \multirow{2}{*}{0.74} \\
\hline $\begin{array}{ll}\text { Tu. multifocality } & \text { single } \\
& \text { multiple (2-7) }\end{array}$ & $30(55.5 \%)$ & $20(54.0 \%)$ & $10(58.8 \%)$ & \\
\hline \multirow{2}{*}{$\begin{array}{ll}\text { Grade } & \text { G1 } \\
& \text { G2 } \\
\end{array}$} & $22(40.7 \%)$ & $16(43.2 \%)$ & $6(35.3 \%)$ & \multirow{2}{*}{0.58} \\
\hline & $32(59.3 \%)$ & $21(56.7 \%)$ & $11(64.7)$ & \\
\hline Recurrence & $8(15.8 \%)$ & $4(10.8 \%)$ & $4(23.5 \%)$ & 0.22 \\
\hline Progression & $2(3.7 \%)$ & 0 & $2(11.7 \%)$ & 0.03 \\
\hline Deaths & $18(33.3 \%)$ & $11(29.7 \%)$ & $7(41.2 \%)$ & 0.40 \\
\hline
\end{tabular}

tients from the high NLR group had recurrence. Multivariable Cox regression analyses proved NLR as prognostic factor regarding recurrence ( $\mathrm{HR}=1.09, \mathrm{p}=0.01)$, the addition of NLR to the standard model (age, gender, diameter, grade and multifocality) increased the Harrel's C index from 73.61 to 83.33. (Table 2). A Kaplan Meier analysis of Recurrence-free survival (RFS) did not show statistical significance among NLR groups: $82.67 \%$ vs. $64.12 \%, p=0.26$. In multivariate Cox regression analysis, NLR as a con- tinuous variable was an independent prognostic factor for RFS (Figure 2).

During follow-up (106 months), 18 patients died, $7(41.2 \%)$ of them belonging to the high NLR group. The Kaplan-Meier OS curve showed a total survival of $70.27 \%$ at 10 years in the low NLR group, whereas the high NLR group demonstrated a $58.82 \%$ OS rate, $\mathrm{p}=0.45$ (Figure $3)$. In the multivariable analysis, the age at the moment of diagnosis was a predictive factor and NLR did not retain a statistically significant as-

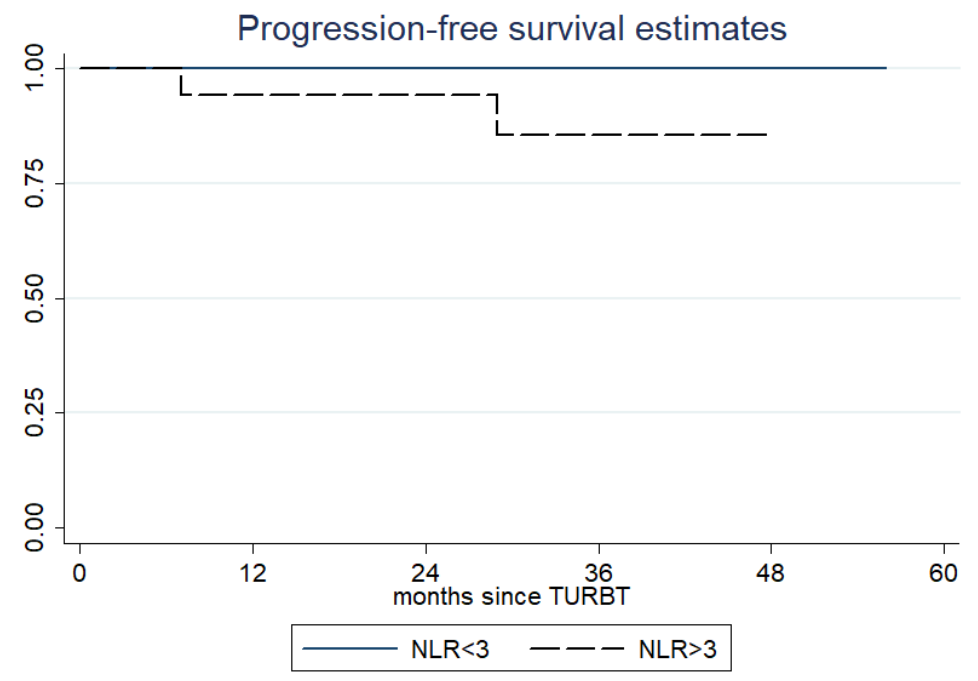

Fig. 1. Progression-free survival estimates of 54 patients with pTa bladder cancer 
Table 2. Multivariable Cox regression analyses predicting recurrence of 54 patients with pTa bladder cancer

\begin{tabular}{lccc}
\hline \multirow{2}{*}{ Prognostic factor } & \multicolumn{3}{c}{ Multivariable } \\
\cline { 2 - 4 } & HR & $\mathbf{9 5} \%$ CI & p value \\
\hline NLR & 1.09 & $1.02-1.16$ & $\mathbf{0 . 0 1}$ \\
\hline Gender Male vs Female & 0.99 & $0.17-5.58$ & 0.99 \\
\hline Age & 0.98 & $0.91-1.06$ & 0.73 \\
\hline Diameter $<3 \mathrm{~cm}$ vs $>3 \mathrm{~cm}$ & 8.23 & $0.77-87.45$ & 0.08 \\
\hline Grade G1 vs G2 & 0.17 & $0.02-1.01$ & 0.052 \\
\hline Multifocality Single vs Multiple & 0.35 & $0.04-3.01$ & 0.34 \\
\hline C-index without NLR (95\%CI) & \multicolumn{3}{c}{$73.61(51.9-98.7)$} \\
\hline C-index with NLR $(95 \% \mathrm{CI})$ & \multicolumn{3}{c}{$83.33(70.45-98.8)$} \\
\hline
\end{tabular}

CI: confidence interval, HR: hazard ratio; *NLR cat: HR 4.81, $95 \%$ CI: $0.86-26.72, p$ value $: 0.07$

sociation (HR 1.03, CI 0.98-1.08, $\mathrm{p}=0.22$ ), however the addition of NLR to the standard model (age, gender, diameter, grade and multifocality) increased the Harrel's C index from 64.84 to 68.05 (Table 3).

\section{Discussion}

The use of biomarkers with predictive and prognostic roles is essential in determining the evolution of tumors. The integration of these markers in daily practice is of utmost importance. With this said, these must be both easy to access and cost-effective (25).

From this point of view, we demonstrated that NLR as a continuous variable is a prognostic factor for recurrence in $\mathrm{pTa}$ NMBIC. As a categorical variable, NLR $>3$ was not statistically significant (HR 4.81, $\mathrm{p}=0.007$ ). It is, however, important for clinical practice to have a reference value, and as NLR $>3$ was validated for high-risk NMBIC (26), this can be used as a cut-off value for low-risk NMIBC. Even more, adding NLR to the standard predictive model for recurrence

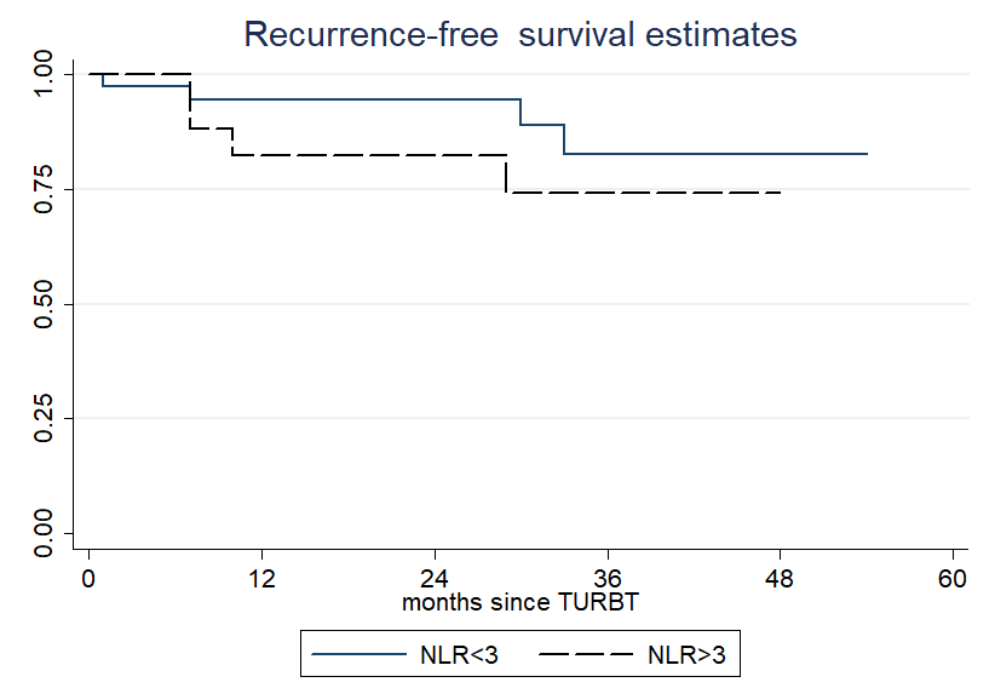

Fig. 2. Recurrence-free survival estimates of 54 patients with pTa bladder cancer 


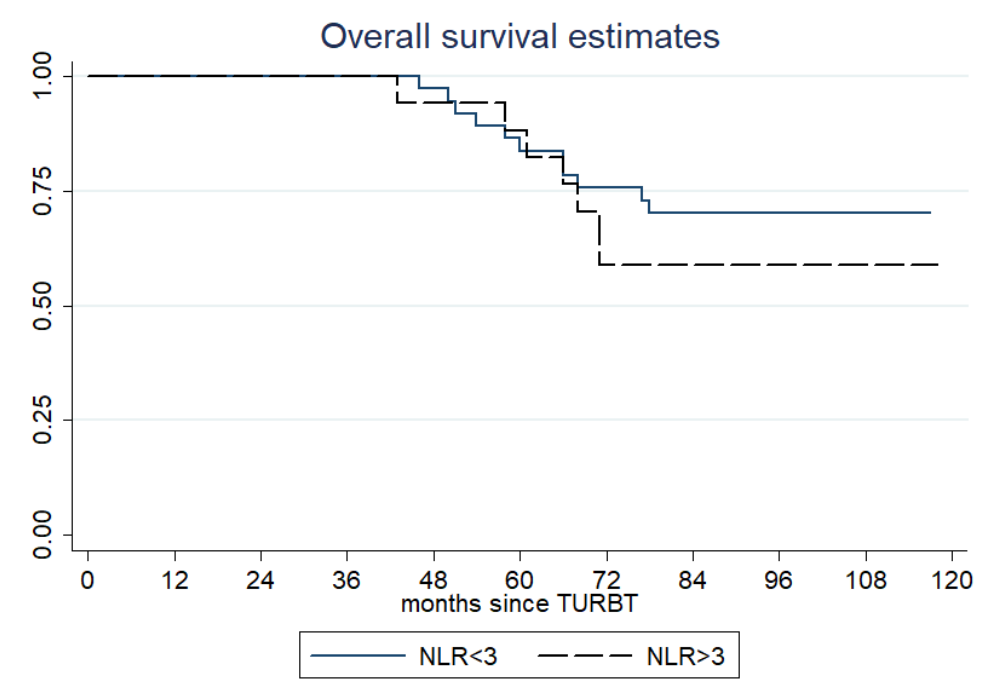

Fig. 3. Overall survival estimates of 54 patients with pTa bladder cancer

increases the $\mathrm{C}$-index by almost 10 points, suggesting that could be a reliable biomarker for clinical decision making in this subgroup of patients with NMIBC.

In terms of survival, the Kaplan-Meier curve showed a total survival of $70.27 \%$ at 10 years in the low NLR group, whereas the high NLR group demonstrated a $58.82 \%$ OS rate. However, there was not a statistically significant difference according to NLR as was shown also in other prospective trials that included MIBC patients (27). Thus, survival was similar to other reports regarding Ta low grade bladder tumors (28).

In the most recent meta-analysis (19) investigating the role of the NLR in the target group of NMIBC undergoing TURBT, it was demonstrated that elevated NLR was associated with reduced RFS and PFS when the NMIBC had previously received BCG (Bacillus Calmette Guerin) instillation. High NLR may be associated with a high risk of recurrence and aggressiveness of the disease in patients who underwent TURBT. Furthermore, this study concluded that there is a lack of data regarding the importance of NLR in Ta NMBIC.

Table 3. Multivariable Cox regression analyses predicting survival of 54 patients with pTa bladder cancer

\begin{tabular}{lccc}
\hline \multirow{2}{*}{ Prognostic factor } & \multicolumn{3}{c}{ Multivariable } \\
\cline { 2 - 4 } NLR & HR & $\mathbf{9 5 \% \text { CI }}$ & p value \\
\hline Gender Male vs Female & 1.03 & $0.98-1.08$ & 0.22 \\
\hline Age & 1.02 & $0.32-3.25$ & 0.96 \\
\hline Diameter $<3 \mathrm{~cm}$ vs $>3 \mathrm{~cm}$ & 1.06 & $1-1.12$ & 0.03 \\
\hline Grade G1 vs G2 & 1.8 & $0.42-7.7$ & 0.42 \\
\hline Multifocality Single vs Multiple & 1.02 & $0.35-2.98$ & 0.96 \\
\hline C-index without NLR $(95 \% \mathrm{CI})$ & 0.72 & $0.18-2.85$ & 0.64 \\
\hline C-index with NLR $(95 \% \mathrm{CI})$ & \multicolumn{3}{c}{$64.84(51.88-77.79)$} \\
\hline
\end{tabular}

CI: confidence interval, HR: hazard ratio; *NLR cat: HR 1.17, $95 \%$ CI: 0.42-3.24, p value : 0.76 
In another meta-analysis that studied patients who had undergone Radical Cystectomy (RC), Lucca et al. (16) highlighted that the preoperatively determined NLR values of 2.7 or higher exhibited statistical significance with advanced tumor stage, lymph node metastases, lympho-vascular system involvement and positive surgical wind. Determination of NLR values contributes, and facilitates the need for further testing and clinical decision-making on treatment.

Several factors can influence the prognosis of bladder carcinomas. The survival rate and prognosis of tumors which are detected and treated at an early stage are better. A retrospective study (29) that investigated the survival rate of Ta stage bladder tumors showed that a later diagnosed bladder cancer has a significantly worse survival rate (69 vs. 59.8 years, $p<0.001$ ). In addition, 10-year survival rate of patients receiving BCG instillations was higher $56.8 \%$. vs $84.4 \%$, p $=0.006$. The survival rates of well-differentiated (G1) and moderately differentiated (G2) Ta stage tumors were proven to be similar (29).

Recurrence and progression of the primary tumor represent a frequent event in the history of NMIBC after TURBT: RFS is higher when a single tumor is found, is smaller than $3 \mathrm{~cm}$, the patient is female, and is G1/G2. However, PFS is not dependent on the number and size of tumors in patients who received BCG bladder instillation after TURBT. A better prognosis and lower recurrence rate was observed (29).

In recent years, great importance has been placed on the prognostic role of blood-based biomarkers in determining the evolution/outcome of tumors. A study published in 2016 (30) highlighted the importance of a preoperatively determined NLR as a potential predictor of the pathological stage of BC. In the study, the 222 patients with $\mathrm{BC}$ all underwent TURBT, and each had a tumor diameter greater than $3 \mathrm{~cm}$. According to the pathological studies, 162 pa- tients had NMIBC, while 60 cases had MIBC. Comparing the degree of tumor coverage with the previously measured laboratory values, the NLR showed a statistically significant difference between the two groups (3.44 vs. $4.65, \mathrm{p}$ $=0.005$ ). In a study involving 107 patients, the prognostic role of NLR in transurethral resection was studied in NMIBC patients, with the NLR cut-off being 2.41. In 68 cases, NLR was higher than 2.41 and when this was the case, the male population was significantly more affected $(p$ $=0.02)$ and $\mathrm{T} 1$ tumors were more frequent $(\mathrm{p}$ $=0.034)$. A higher NLR value showed a slight positive relationship with the higher degree of differentiation $(\mathrm{R}=0.21, \mathrm{P}=0.028)$. The threeyear PFS rate was significantly higher in those with lower NLR (NLR $\leq 2.41,84 \%, p=0.004)$. RFS was also significantly higher in patients with lower NLR (27\% vs. 56\%, p = 0.016) (31). The role of other inflammatory markers in the prognosis of bladder tumors has also been demonstrated. Schulz et al. (32) investigated the inflammatory factors found in the blood in the oncological outcome of BC. In their observations, the lower platelet-lymphocyte ratio $(P L R \leq 28)$ and leukocytosis were found to be significant with adverse oncological outbreaks. Advanced BC occurred more frequently in patients with low PLR (pT2-4 (35\%) vs. pT $\leq 1$ $(24 \%) \mathrm{p}=0.006)$ and leukocytosis (pT2-4 (46\%) vs. pT $\leq 1(30 \%)$ p $<0.047)$. A meta-analysis (33) performed in 2014 highlights the role of C-reactive protein in the outcome of urological neoplasms with a negative impact on RFS and OS. In Gierth et al.'s (34) 2015 study, the investigators reported that preoperative anemia in patients with $\mathrm{BC}$ who underwent $\mathrm{RC}$ worsened the prognosis for the outcome of the disease.

Limitations of this study include its retrospective nature; however, we followed patients for almost 10 years after TURBT. Additionally, sample size was another limitation, but if we consider the 
long follow-up and previous results we reported (35) for high-grade NMIBC (those that also came from a mono-center cohort and that were further validated in a multi-institutional cohort (23)), we can consider our results reliable. Of course, these findings must be externally validated in a multi-institutional study.

\section{Conclusion}

In this cohort, high NLR was associated with a higher rate of recurrence in patients with $\mathrm{pTa}$ NMIBC. Further prospective and multi-center studies should focus on validating these findings, as in this particular sub-group of patients with bladder cancer NLR could represent a valid biomarker for clinical usage regarding the intensity of follow-up schedule.

\section{Abbreviations}

BC - bladder cancer

NLR - neutrophil-to-lymphocyte ratio

NMIBC - non-muscle invasive bladder cancer

TURBT - transurethral resection of bladder tumor

IQR - interquartile range

EORTC - European Organization of Research and Treatment of Cancer

RFS - recurrence-free survival

PFS - progression-free survival

OS - overall survival

CIS - in situ carcinoma

MIBC - muscle-invasive bladder cancer

$\mathrm{HG}$ - high-grade

LG - low-grade

EAU - European Association of Urology

BCG - Bacillus Calmette Guerin

$\mathrm{RC}$ - radical cystectomy

PLR - platelet-lymphocyte ratio

$\mathrm{p} \mathrm{Ta}$ - pathological assesment of the primary tumor

\section{Authors Contribution}

MO - Conceptualization, Validation, Supervision, Resources, Project Administration

BD - Methodology, Writing, Visualization

PHD - Methodology, Validation, Software

DE - Writing, Data Curation, Investigation

VMD - Methodology, Writing, Software, Formal Analysis, Data Curation

CBC - Validation, Visualization, Software

BA - Resources, Validation, Visualization

PA - Validation, Formal Analysis, Software

VAO - Conceptualization, Supervision, Visualization

\section{Conflict of interest}

None to declare.

\section{References}

1. Bray F, Ferlay J, Soerjomataram I, Siegel RL, Torre LA, Jemal A. Global cancer statistics 2018: GLOBOCAN estimates of incidence and mortality worldwide for 36 cancers in 185 countries. CA: A Cancer Journal for Clinicians. 2018;68(6):394-424. DOI: 10.3322/ caac. 21492

2. Wong MCS, Fung FDH, Leung C, Cheung WWL, Goggins $\mathrm{WB}, \mathrm{Ng} \mathrm{CF}$. The global epidemiology of bladder cancer: a joinpoint regression analysis of its incidence and mortality trends and projection. Sci Rep. 2018 18;8(1):1129. DOI: 10.1038/s41598-018-19199-z

3. Sylvester RJ, van der Meijden APM, Oosterlinck W, Witjes JA, Bouffioux C, Denis L, et al. Predicting recurrence and progression in individual patients with stage Ta T1 bladder cancer using EORTC risk tables: a combined analysis of 2596 patients from seven EORTC trials. Eur Urol. 2006 Mar;49(3):466-465; discussion 475-477. DOI: 10.1016/j.eururo.2005.12.031

4. Fernandez-Gomez J, Madero R, Solsona E, Unda M, Martinez-Pi-eiro L, Gonzalez M, et al. Predicting nonmuscle invasive bladder cancer recurrence and progression in patients treated with bacillus Calmette-Guerin: the CUETO scoring model. J Urol. 2009 Nov;182(5):2195-203. DOI: 10.1016/j. juro.2009.07.016

5. Rayn KN, Hale GR, Grave GP-L, Agarwal PK. New therapies in nonmuscle invasive bladder cancer treatment. Indian J Urol. 2018 Mar;34(1):11-9. DOI: 10.4103/iju.IJU_296_17

6. Isharwal S, Konety B. Non-muscle invasive blad- 
der cancer risk stratification. Indian J Urol. 2015 Dec;31(4):289-96. DOI: 10.4103/0970-1591.166445

7. Veeratterapillay R, Heer R, Johnson MI, Persad R, Bach C. High-Risk Non-Muscle-Invasive Bladder Cancer-Therapy Options During Intravesical BCG Shortage. Curr Urol Rep. 2016 Sep;17(9):68. DOI: 10.1007/ s 11934-016-0625-z

8. Konety BR, Williams RD. Superficial transitional (Ta/T1/CIS) cell carcinoma of the bladder. BJU Int. 2004 Jul;94(1):18-21 . DOI: 10.1111/j.1464410X.2003.04894.x

9. Rouprêt M, Seisen T, Compérat E, Larré S, Mazerolles $\mathrm{C}$, Gobet $\mathrm{F}$, et al. Prognostic interest in discriminating muscularis mucosa invasion (T1a vs T1b) in nonmuscle invasive bladder carcinoma: French national multicenter study with central pathology review. J Urol. 2013 Jun;189(6):2069-76. DOI: 10.1016/j.juro.2012.11.120

10. Baranyi M, Lippai M, Szatmári Z. [Role of the stro$\mathrm{ma}$ in the initiation and progression of tumors]. Orv Hetil. 2015 Nov 8;156(45):1816-23. DOI: 10.1556/650.2015.30294

11. Vartolomei MD, Mathieu R, Margulis V, Karam JA, Rouprêt M, Lucca I, et al. Promising role of preoperative neutrophil-to-lymphocyte ratio in patients treated with radical nephroureterectomy. World J Urol. 2017 Jan;35(1):121-30. DOI: 10.1007/s00345-016-1848-9

12. Stotz M, Gerger A, Eisner F, Szkandera J, Loibner H, Ress AL, et al. Increased neutrophil-lymphocyte ratio is a poor prognostic factor in patients with primary operable and inoperable pancreatic cancer. Br J Cancer. 2013 Jul 23;109(2):416-21. DOI: 10.1038/bjc.2013.332

13. de Martino M, Pantuck AJ, Hofbauer S, Waldert M, Shariat SF, Belldegrun AS, et al. Prognostic impact of preoperative neutrophil-to-lymphocyte ratio in localized nonclear cell renal cell carcinoma. J Urol. 2013 Dec;190(6):1999-2004. DOI: 10.1016/j. juro.2013.06.082

14. Mbeutcha A, Rouprêt M, Kamat AM, Karakiewicz PI, Lawrentschuk N, Novara G, et al. Prognostic factors and predictive tools for upper tract urothelial carcinoma: a systematic review. World J Urol. 2017 Mar;35(3):33753. DOI: $10.1007 / \mathrm{s} 00345-016-1826-2$

15. Mathieu R, Vartolomei MD, Mbeutcha A, Karakiewicz PI, Briganti A, Roupret M, et al. Urothelial cancer of the upper urinary tract: emerging biomarkers and integrative models for risk stratification. Minerva Urol Nefrol. 2016 Aug;68(4):381-95.

16. Lucca I, Jichlinski P, Shariat SF, Rouprêt M, Rieken M, Kluth LA, et al. The Neutrophil-to-lymphocyte Ratio as a Prognostic Factor for Patients with Urothelial Carcinoma of the Bladder Following Radical Cystectomy: Validation and Meta-analysis. Eur Urol Focus. 2016 Apr;2(1):79-85. DOI: 10.1016/j.euf.2015.03.001

17. Tang X, Du P, Yang Y. The clinical use of neutrophil-to-lymphocyte ratio in bladder cancer patients: a systematic review and meta-analysis. Int J Clin Oncol. 2017 Oct;22(5):817-25. DOI: 10.1007/s10147-0171171-5

18. Rouprêt M, Babjuk M, Compérat E, Zigeuner R, Sylvester RJ, Burger M, et al. European Association of Urology Guidelines on Upper Urinary Tract Urothelial Carcinoma: 2017 Update. Eur Urol. 2018;73(1):11122. DOI: $10.1016 /$ j.eururo.2017.07.036

19. Vartolomei MD, Porav-Hodade D, Ferro M, Mathieu R, Abufaraj M, Foerster B, et al. Prognostic role of pretreatment neutrophil-to-lymphocyte ratio (NLR) in patients with non-muscle-invasive bladder cancer (NMIBC): A systematic review and meta-analysis. Urol Oncol. 2018;36(9):389-99. DOI: 10.1016/j.urolonc.2018.05.014

20. Montironi R, Lopez-Beltran A. The 2004 WHO classification of bladder tumors: a summary and commentary. Int J Surg Pathol. 2005 Apr;13(2):143-53. DOI: $10.1177 / 106689690501300203$

21. Babjuk M, Oosterlinck W, Sylvester R, Kaasinen E, Böhle A, Palou-Redorta J, et al. EAU guidelines on non-muscle-invasive urothelial carcinoma of the bladder. Eur Urol. 2008 Aug;54(2):303-14. DOI: 10.1016/j. eururo.2008.04.051

22. Babjuk M, Böhle A, Burger M, Capoun O, Cohen D, Compérat EM, et al. EAU Guidelines on Non-Muscle-invasive Urothelial Carcinoma of the Bladder: Update 2016. Eur Urol. 2017;71(3):447-61. DOI: 10.1016/j.eururo.2016.05.041

23. Woldu SL, Bagrodia A, Lotan Y. Guideline of guidelines: non-muscle-invasive bladder cancer. BJU Int. 2017;119(3):371-80. DOI: 10.1111/bju. 13760

24. Newson, R. B. (2010). Comparing the Predictive Powers of Survival Models Using Harrell's C or Somers' D. The Stata Journal, 10(3), 339-358. DOI: $10.1177 / 1536867 X 1001000303$

25. Mathieu R, Lucca I, Rouprêt M, Briganti A, Shariat SF. The prognostic role of lymphovascular invasion in urothelial carcinoma of the bladder. Nat Rev Urol. 2016;13(8):471-9. DOI: 10.1038/nrurol.2016.126

26. Vartolomei MD, Ferro M, Cantiello F, Lucarelli G, Di Stasi S, Hurle R, et al. Validation of Neutrophil-to-lymphocyte Ratio in a Multi-institutional Cohort of Patients With T1G3 Non-muscle-invasive Bladder Cancer. Clin Genitourin Cancer. 2018;16(6):445-52. DOI: 10.1016/j.clgc.2018.07.003

27. Ojerholm E, Smith A, Hwang W-T, Baumann BC, Tucker KN, Lerner SP, et al. Neutrophil-to-lymphocyte ratio as a bladder cancer biomarker: Assessing prognostic and predictive value in SWOG 8710. Cancer. 2017 Mar 1;123(5):794-801. DOI: 10.1002/cncr.30422

28. Vollmer RT. A Review of Outcomes for Stage Ta Bladder Tumors. Am J Clin Pathol. 2016 Aug;146(2):21520. DOI: $10.1093 /$ ajcp/aqw103

29. Balan D, Martha O, Chibelean CB, Tataru S, Voidezan 
$\mathrm{S}$, Sin A, et al. Comparison of 10-year overall survival between patients with G1 and G2 grade Ta bladder tumors. Medicine (Baltimore). 2018 Apr;97(16):e0522. DOI: 10.1097/MD.0000000000010522

30. Celik O, Akand M, Keskin MZ, Yoldas M, Ilbey YO. Preoperative neutrophil-to-lymphocyte ratio (NLR) may be predictive of pathologic stage in patients with bladder cancer larger than $3 \mathrm{~cm}$. Eur Rev Med Pharmacol Sci. 2016;20(4):652-6.

31. Mano R, Baniel J, Shoshany O, Margel D, Bar-On T, Nativ O, et al. Neutrophil-to-lymphocyte ratio predicts progression and recurrence of non-muscle-invasive bladder cancer. Urol Oncol. 2015 Feb;33(2):67.e1-7. DOI: 10.1016/j.urolonc.2014.06.010

32. Schulz GB, Grimm T, Buchner A, Jokisch F, Grabbert M, Schneevoigt B-S, et al. Prognostic Value of the Preoperative Platelet-to-leukocyte Ratio for Oncologic Outcomes in Patients Undergoing Radical Cystectomy for Bladder Cancer. Clin Genitourin Cancer. 2017;15(6):e915-21. DOI: 10.1016/j.clgc.2017.05.009

33. Dai J, Tang K, Xiao W, Yu G, Zeng J, Li W, et al. Prognostic significance of C-reactive protein in urological cancers: a systematic review and meta-analysis. Asian Pac J Cancer Prev. 2014;15(8):3369-75. DOI: 10.7314/ APJCP.2014.15.8.3369

34. Gierth M, Mayr R, Aziz A, Krieger S, Wullich B, Pycha A, et al. Preoperative anemia is associated with adverse outcome in patients with urothelial carcinoma of the bladder following radical cystectomy. J Cancer Res Clin Oncol. 2015 Oct;141(10):1819-26. DOI: 10.1007/ s00432-015-1957-7

35. Martha O, Porav-Hodade D, Bălan D, Tătaru OS, Sin A, Chibelean CB, et al. Easily Available Blood Test Neutrophil-To-Lymphocyte Ratio Predicts Progression in High-Risk Non-Muscle Invasive Bladder Cancer. Rev Romana Med Lab. 2017;25(2):181-9. DOI: 10.1515/ rrlm-2017-0016 